\title{
CAPILLARY PHENOMENA ON A LIQUID SURFACE
}

\author{
Mohammad Ali and Akira Umemura \\ Department of Aerospace Engineering, Graduate School of Engineering, Nagoya University
}

Furo-cho, Chikusa-ku, Nagoya 464-8603, Japan

\begin{abstract}
Contraction of a liquid sheet of an incompressible Newtonian fluid in a passive ambient fluid is studied computationally to provide insights into the dynamics of capillary wave created during contraction. The problem composed of the Navier-Stokes system is associated with initial and boundary conditions that govern the time evolution of the capillary wave and the pressure and velocity fields within it. The correctness of the algorithm is verified with the data of experiment. It can be found that the prediction of the computation agrees well with the experiment. The algorithm is capable of capturing the capillary wave and therefore it is used to study the characteristic phenomena of that wave created on the surface of the liquid. Results show that the capillary wave is radiated from the tip of the liquid sheet caused by surface tension. The amplitude of the tip wave is much larger than any other waves and the asymptotic approach of the wave peaks can be observed during the propagation of waves. The tip wave contains the highest pressure and gradually the peak values of both high and low pressures decrease with the propagation of waves. Fluid velocity is motivated by both pressure due to surface tension and recirculation in peak and trough of the wave. During the contraction of the liquid sheet, the tip velocity is not uniform. Initially the length of the sheet increases a little and very soon the contraction occurs and continues. After long time, the gradient of tip velocity becomes very small.
\end{abstract}

Keywords: Capillary wave, liquid sheet.

\section{INTRODUCTION}

There are many industrial processes where liquid spray is widely used in the system and an important factor for producing quality products. The purpose of liquid breaking in a spray is to increase the liquid surface area for increasing the subsequent heat and mass transfer. The spatial distribution, or dispersion of the droplets, is important in many combustion systems because it affects the mixing of fuel with oxidizer, which influences the flame length. Therefore to understand the mechanism of atomization is very important for its efficient application and to improve the design of practical combustion devices. Before occurring atomization, a wave of very small amplitude is formed on the liquid surface, which is called capillary wave. The detailed knowledge on the characteristics of capillary wave is required to understand the insight mechanisms for the disintegration of liquid. However, it is a difficult task to capture the capillary wave in numerical simulation. A suitable numerical algorithm is required to solve the problem. Several methods were proposed and in use for the simulation of such flow problems. These methods are discussed in reference ${ }^{1-4}$. Gueyffier et al. ${ }^{1}$ described a numerical scheme for interface calculations. The author used the volume of fluid interface tracking method and a piecewise linear interface calculation in the scheme. The method of interface tracking with the connection of volume fraction and interface position was described in detail. A new model called continuum surface force (CSF) for surface tension effects on fluid motion was developed by Brackbill et al. ${ }^{2}$. The model interpreted the surface tension as a continuous, three-dimensional effect across an interface, rather than as a boundary value condition on the interface. Hirt et $\mathrm{al}^{3}{ }^{3}$ made a short review of different methods used for embedding free boundaries and compared the relative advantages and disadvantages of these methods. The author introduced a new technique in the volume of fluid (VOF) method which worked well for complicated problems.
Welch et al. ${ }^{4}$ used a VOF based interface tracking method in conjunction with a mass transfer model and used in simulation of horizontal film boiling problem. A new algorithm for the volume tracking of interfaces in twodimensions was proposed by Rider and Kothe ${ }^{5}$. The method utilized local discrete material volume and velocity data to track liquid interfaces. A Flux Line-Segment model for two-dimensional problem was employed by Ashgriz and $\mathrm{Poo}^{6}$ for tracking the interfaces. All the above researchers used VOF method for two-phase flow problems with the conjunction of some other models and techniques. Therefore, it can be concluded that the VOF method is one of the most popular schemes for tracking interfaces and hence implemented in present algorithm. The section- 3 of this paper describes the VOF technique a little in detail.

In liquid jet atomization, capillary instability and disintegration of liquid are important and interesting phenomena to the fluid dynamists. An earlier account of the work is summarized by Rayleigh ${ }^{7}$ who performed a delightful discussion on jet instability and published both theoretical and experimental results on capillary instability phenomena. During the contraction of liquid jet or cylindrical liquid drop the capillary waves are radiated on the fluid interfaces. These waves are caused by surface tension and generated at the tip of liquid jet. In an experiment Goedde and Yuen $^{8}$ examined the capillary instability of vertical liquid jets of different viscosities and measured the growth rates of waves for disturbances of various wavelengths. The author discussed about the drop formation and ligament detachment and showed that some non-linear effects became very pronounced especially at small wave number and dominated the growth processes. In another investigation Donnelly and Glaberson ${ }^{9}$ performed some experiments and discussed the effects of viscosity on the capillary instability and growth rate. Also several other investigations ${ }^{10}, 11$ can be found in the literature which described general features on end pinching

Journal of Mechanical Engineering, vol. ME38, Dec. 2007

Transaction of the Mech. Eng. Div., The Institution of Engineers, Bangladesh 
of elongated liquid drops, their deformation and breakup. In these investigations the authors discussed and explained the effects of initial drop shape and the relative viscosities of the two fluids on the relaxation and breakup phenomena. No capillary wave was observed at the tip of the elongated liquid drop with the conditions they considered. Obviously, the phenomenon of capillary wave is a complicated one and difficulties are there to capture this wave in the resolution.

In an investigation of axisymmetric free-surface flow, Eggers $^{12}$ searched the characteristic behaviour of drop formation from a liquid column and found that the breaking of fluid neck was independent of the microscopic structure of the fluid, rather it was hydrodynamic phenomena. To search the dynamics in the immediate viscinity of the point of liquid breakup, Eggers ${ }^{13}$ performed another study on theory of drop formation by analyzing the motion of liquid column using Navier-Stokes equations. The author found an asymptotic solution for both before and after singularity of breaking point. After the singularity, the fluid consisted of two halves, which constituted a unique continuation of the Navier-Stokes equation through the singular point. The nature of the singularities was also investigated in detail by Eggers and Dupont ${ }^{14}$ using a one-dimensional equation of motion derived from the Navier-Stokes equations for the velocity and the radius during the formation of drop. In an experimental investigation, Kowalewski ${ }^{15}$ studied the details of the shape of the thin liquid neck joining the droplet to its parent body in terms of the fluid viscosity and its jet diameter. The author showed that initially the tip of the jet close to the droplet became convergent, elongated and formed a structure called macro-thread. The tip of the jet began to become thin and created the second, thin micro-thread between the macro-thread and the droplet. It was found that the maximum length of micro-thread was strongly dependent on liquid viscosity and the retraction velocity after the breakup exhibited relatively small variation with viscosity. An elaborated review work on the drop formation and breakup dynamics was performed by Eggers ${ }^{16}$ and outlined some unresolved problems on it. The author discussed both the theoretical development as well as the experimental investigations on this field. The review work was motivated by the expectation that the pinching was universal under quite general circumstances, even if the motion farther away from the singularity was more complicated. In the non-asymptotic regime, the author focused on the axisymmetric case of a jet with or without gravity which excluded nonlinear free-surface motion, such as drop oscillations, the dynamics of fluid sheets, and in particular the vast field of surface waves.

From the above discussion, it is revealed that the study of the capillary wave created on the liquid surface has been given little attention in the literature, which has been addressed in the present study. To achieve the goal, a three-dimensional numerical code is developed and validated with experimental data. The code is then used for numerical simulation to understand the phenomena of capillary waves radiated from the tip of the liquid sheet in an otherwise quiescent fluid.

\section{MATHEMATICAL MODELING}

The flow field is governed by time dependent threedimensional Navier-Stokes equations with surface tension force. Body forces are neglected. These equations can be expressed as

$$
\begin{aligned}
& \frac{\partial \overrightarrow{\mathrm{U}}}{\partial \mathrm{t}}+\frac{\partial \overrightarrow{\mathrm{P}}}{\partial \mathrm{x}}+\frac{\partial \overrightarrow{\mathrm{Q}}}{\partial \mathrm{y}}+\frac{\partial \overrightarrow{\mathrm{R}}}{\partial \mathrm{z}}=\frac{\partial \overrightarrow{\mathrm{P}}_{\mathrm{v}}}{\partial \mathrm{x}}+\frac{\partial \overrightarrow{\mathrm{Q}}_{\mathrm{v}}}{\partial \mathrm{y}}+\frac{\partial \overrightarrow{\mathrm{R}}_{\mathrm{v}}}{\partial \mathrm{z}}+\overrightarrow{\mathrm{F}}_{\mathrm{sv}} \\
& \mathrm{U}=\left(\begin{array}{l}
\rho \\
\rho \mathrm{u} \\
\rho \mathrm{v} \\
\rho \mathrm{w}
\end{array}\right), \quad \mathrm{P}_{\mathrm{v}}=\left(\begin{array}{l}
0 \\
\tau_{\mathrm{xx}} \\
\tau_{\mathrm{xy}} \\
\tau_{\mathrm{zx}}
\end{array}\right), \quad \mathrm{P}=\left(\begin{array}{l}
\rho \mathrm{u} \\
\rho \mathrm{u}^{2}+\mathrm{p} \\
\rho \mathrm{uv} \\
\rho \mathrm{uw}
\end{array}\right), \quad \mathrm{Q}=\left(\begin{array}{l}
\rho \mathrm{v} \\
\rho \mathrm{uv} \\
\rho v^{2}+\mathrm{p} \\
\rho \mathrm{w}
\end{array}\right), \\
& \mathrm{Q}_{\mathrm{v}}=\left(\begin{array}{l}
0 \\
\tau_{\mathrm{xy}} \\
\tau_{\mathrm{yy}} \\
\tau_{\mathrm{yz}}
\end{array}\right), \quad \mathrm{R}=\left(\begin{array}{l}
\rho \mathrm{w} \\
\rho \mathrm{uw} \\
\rho \mathrm{vw} \\
\rho \mathrm{w}^{2}+\mathrm{p}
\end{array}\right), \mathrm{R}_{\mathrm{v}}=\left(\begin{array}{l}
0 \\
\tau_{\mathrm{zx}} \\
\tau_{\mathrm{yz}} \\
\tau_{\mathrm{zz}}
\end{array}\right), \mathrm{F}_{\mathrm{sv}}=\left(\begin{array}{l}
0 \\
\sigma \kappa \mathrm{f}_{\mathrm{x}} \\
\sigma \kappa \mathrm{n}_{\mathrm{y}} \\
\sigma \kappa \mathrm{n}_{\mathrm{z}}
\end{array}\right)
\end{aligned}
$$

The following terms are expressed as,

$$
\begin{aligned}
& \tau_{\mathrm{xx}}=\lambda\left(\frac{\partial \mathrm{u}}{\partial \mathrm{x}}+\frac{\partial \mathrm{v}}{\partial \mathrm{y}}+\frac{\partial \mathrm{w}}{\partial \mathrm{z}}\right)+2 \mu\left(\frac{\partial \mathrm{u}}{\partial \mathrm{x}}\right) \\
& \tau_{\mathrm{yy}}=\lambda\left(\frac{\partial \mathrm{u}}{\partial \mathrm{x}}+\frac{\partial \mathrm{v}}{\partial \mathrm{y}}+\frac{\partial \mathrm{w}}{\partial \mathrm{z}}\right)+2 \mu\left(\frac{\partial \mathrm{v}}{\partial \mathrm{y}}\right) \\
& \tau_{\mathrm{zz}}=\lambda\left(\frac{\partial \mathrm{u}}{\partial \mathrm{x}}+\frac{\partial \mathrm{v}}{\partial \mathrm{y}}+\frac{\partial \mathrm{w}}{\partial \mathrm{z}}\right)+2 \mu\left(\frac{\partial \mathrm{w}}{\partial \mathrm{z}}\right) \\
& \tau_{\mathrm{xy}}=\mu\left(\frac{\partial \mathrm{u}}{\partial \mathrm{y}}+\frac{\partial \mathrm{v}}{\partial \mathrm{x}}\right), \tau_{\mathrm{yz}}=\mu\left(\frac{\partial \mathrm{w}}{\partial \mathrm{y}}+\frac{\partial \mathrm{v}}{\partial \mathrm{z}}\right), \tau_{\mathrm{zx}}=\mu\left(\frac{\partial \mathrm{w}}{\partial \mathrm{x}}+\frac{\partial \mathrm{u}}{\partial \mathrm{z}}\right) \\
& \lambda=-\frac{2}{3} \mu .
\end{aligned}
$$

Where, $\mathrm{u}, \mathrm{v}, \mathrm{w}$ are velocities in the flow field, $\rho$ the density, $\mathrm{p}$ the pressure, $\mu$ the viscosity, $\mathrm{F}_{\mathrm{sv}}$ the surface tension force, $\sigma$ the surface tension, $\kappa$ the curvature of surface, $\mathrm{n}$ the unit normal to the surface and $\mathrm{f}$ is a function for continuous change of the color variable (here density) across the thickness of fluid interface.

\section{NUMERICAL MODEL}

A suitable computer code is required to investigate the capillary wave phenomena and to understand the underlying physics in the process. For numerical simulation, volume-of-fluid (VOF) method based on a simplified treatment of the Navier-Stokes equations (1) with a fixed, regular, uniform grid is used to solve the problem. Piecewise Linear Interface Calculation (PLIC) is implemented for the advection of the liquid interface. The treatment of surface tension consists of artificially smoothing the discontinuity present at the interface is a Continuum Surface Force (CSF) manner ${ }^{2}$.

\section{Calculation of normal vector}

The construction of algorithm for calculation of normal vector is based on the idea that a normal vector together with the fractional volume of one fluid contained by the cell determines a unique planar surface cutting the cell into two parts as shown in Fig.1. Each part of the cell contains proper volume of one of the two fluids. Figure 1 shows a planar surface $\mathrm{ABCD}$, under which the liquid lies in the cell.

The normal to the fluid planar surface is estimated by the following finite difference formula as

$$
\overrightarrow{\mathrm{q}}=\vec{\nabla} \mathrm{C}
$$

Where, $\vec{q}$ is the normal vector to the planar surface made by the fluid in each cell and $\mathrm{C}$ is the fractional volume of the fluid contained by the cell. More details of normal vector estimation can be found in reference ${ }^{1}$ for two- 


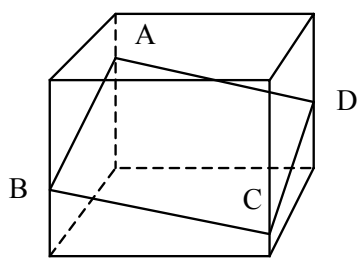

Fig. 1: Liquid planar surface, $A B C D$ cuts the cubic cell into two parts

dimensional case. The procedure can be extended for threedimensional calculations. Once the three components of normal vector are found, the resultant of normal vectors are calculated as

$$
\mathrm{q}=\sqrt{\mathrm{q}_{\mathrm{x}}^{2}+\mathrm{q}_{\mathrm{y}}^{2}+\mathrm{q}_{\mathrm{z}}^{2}}
$$

The components of unit normal vector is calculated as

$$
\mathrm{n}_{\mathrm{x}}=\frac{\mathrm{q}_{\mathrm{x}}}{\mathrm{q}}, \quad \mathrm{n}_{\mathrm{y}}=\frac{\mathrm{q}_{\mathrm{y}}}{\mathrm{q}}, \quad \mathrm{n}_{\mathrm{z}}=\frac{\mathrm{q}_{\mathrm{z}}}{\mathrm{q}}
$$

\section{Calculation of fractional volume and interface position}

To determine the fractional volume and interface position, a parameter is searched which is related to the smallest distance between the planar surface of liquid and the origin of the cell. Therefore this parameter represents the distance along the normal and also defines the planar surface of liquid in the cell. Utilizing this parameter we can determine the area of different sides of the cell occupied by the liquid. A comprehensive description of this calculation can be found in reference ${ }^{1}$ where the description is started with two-dimensions and later it is generalized to three dimensions for calculation of area as well as volume of the fluid. Both area and volume calculations are continuous, one-to-one, and have a functional relationship with volume inside the cell lying below the planar surface and the parameter which characterizes the plane. For easy calculation of area and volume of the liquid, the cells are identified as three categories: (i) cells with zero value of one component of normal to the planar surface, (ii) cells with zero values of two components of normal to the planar surface, and (iii) cells with non-zero normal components. Algorithm is constructed for these categories of cells with the implementation of all possible logics.

\section{Propagation of the interface segments}

After the construction of liquid interface in cell, its motion in the underlying flow field must be introduced in the algorithm by a suitable advection method. Since the atomization process is very fine and rapid, the simulating code should be capable of capturing all the phenomena behind the atomization process. It can be pointed out that the proper and precise computation of this type of problem is a complex one even when it is two-dimensional case. More complexity arises when the problem is three dimensional. Therefore, calculation of fluid flux should be precise for accuracy of the result. By the motion of the fluid, flux of fluid from one cell to its neighboring cells is calculated. Using the expression described in reference ${ }^{1}$ we can determine the "wetted" area occupied by the liquid in the cell through which the flux of fluid occurs. But this area does not remain constant during one time step. To incorporate this change of flux area, first we need to search the cells in which the "wetted" area is changing during the time step and then calculate the changing area as well as flux of the liquid. It can be pointed out that during

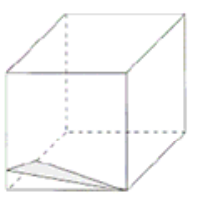

(a)

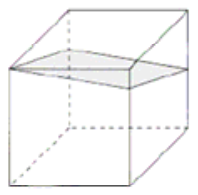

(d)

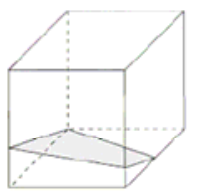

(b)

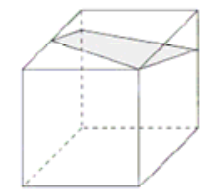

(c)

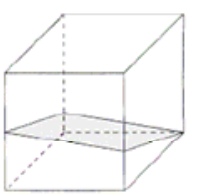

(c)

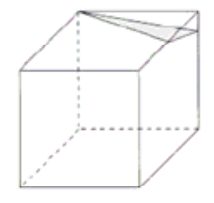

(f)
Fig.2: The different critical shapes of the cut cube ${ }^{1}$. The liquid in the cube changes shape with time and the plane crosses vertices.

calculation, different critical shapes of the cut cube can occur as shown in Fig. 2 where six critical shapes of the cut cubes are presented. Special care must be taken for the advection of liquid from one computational cell to neighboring cells during this occurrence. In present calculation after finding out the cells, the change of flux is calculated with the conjunction of normal to liquid planar surface and geometry of the interface position.

\section{The continuum method}

The interface where the fluid changes from one fluid to the other discontinuously is replaced by a continuous transition. It is not appropriate to apply a pressure jump induced by surface tension at the interface. Rather, the surface tension should act everywhere within the transition region. In fact, the surface tension contributes a surface pressure which is the normal force per unit interfacial area.

In present work the surface tension force is estimated by a volume force which gives the correct surface tension. The volume force is then calculated with the area integral over the portion of the interface lying within the small volume of integration. A suitable color function (density for present investigation) is chosen for smooth variation over the thickness across the interface. A detail description and the formulation for numerical simulation can be found in reference ${ }^{1}$. The model is implemented in the algorithm for present investigation.

\section{RESULTS AND DISCUSSION}

The present investigation can be divided into two parts: Validation of the numerical method and numerical simulation of the contraction of a square cylindrical liquid column to understand the underlying physics for capillary wave instabilities and liquid disintegration processes. To validate the numerical method, a comparison of numerical simulation with published experimental data has been performed. Once the result of comparison is satisfied, the numerical method is applied for the rest part of investigation.

\section{Comparison of numerics with experiment}

The present three-dimensional numerical code is validated by comparing the simulation results with the data of Goedde and Yuen ${ }^{8}$. For comparison a square cylindrical water column is considered to calculate the swell and neck diameters of the capillary wave during its relaxation. The unperturbed cross sectional area of the column and other 


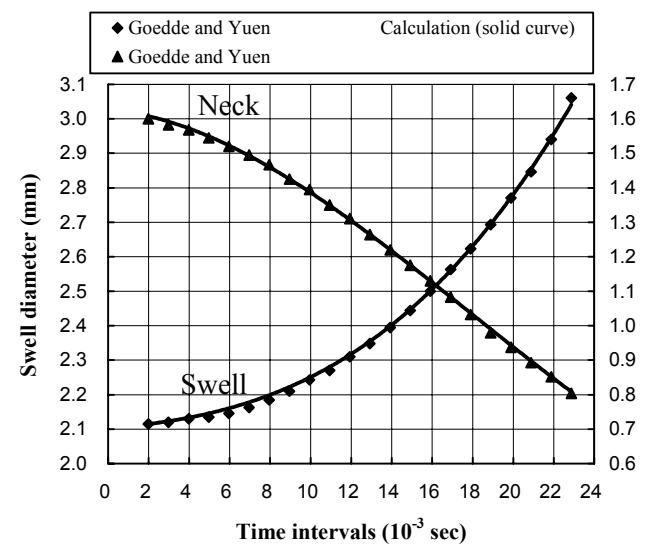

Fig. 3: Comparison of swell and neck diameters with experiment; Average diameter of unperturbed cylinder, $\mathrm{D}_{0}=1.86 \mathrm{~mm}$

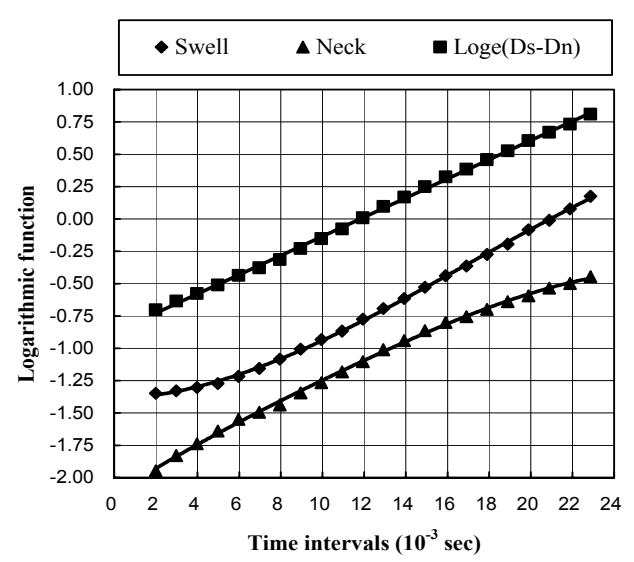

Fig. 4: Logarithmic plot of calculation in Fig. 3 as a function of time. Ds denotes diameter of swell and Dn diameter of neck. Symbol $\bullet$ is for $\log _{e}\left[\operatorname{Ds}(\mathrm{t})-\mathrm{D}_{0}\right] ; \boldsymbol{\Lambda}$ for $\log _{\mathrm{e}}\left[\mathrm{D}_{0}-\mathrm{Dn}(\mathrm{t})\right]$; $\boldsymbol{\boldsymbol { }}$ for $\log _{\mathrm{e}}[\operatorname{Ds}(\mathrm{t})-\mathrm{Dn}(\mathrm{t})]$. The curve of $\log _{\mathrm{e}}\left[\mathrm{D}_{0}-\mathrm{Dn}(\mathrm{t})\right]$ has been shifted downward by a scale reading of 0.5 .

calculation parameters such as surface tension, viscosity etc. are identical with the experiment. The time evolution of both swell and neck during numerical simulation and experiment is shown in Fig. 3. It can be pointed out that during relaxation of liquid cylinder the capillary wave is initiated from tip of the cylinder by surface tension. Due to the shape of cylinder tip the initial growth of the first swell is rapid. Therefore, for comparison the growth of second swell is calculated which is closer to the experimental conditions from geometric point of view. As data taking manner of Goedde and Yuen ${ }^{8}$, the growth of adjacent neck ahead of the swell is considered. For this comparison smoothed experimental data are taken from Fig. 5 of Goedde and Yuen ${ }^{8}$. Figure 3 shows that in general the simulation results agree quite well with the experiment. At the beginning of time evolution history very small deviation from data for both swell and neck diameter can be found. This is caused by the initial shape of the liquid column.
Figure 4 shows the re-plotting of the simulation data in Fig. 3. The Rayleigh's linearized analysis shows that the exponential growth rate is constant. Therefore, the natural logarithms of the deformations of swell, neck and the difference between the swell and neck diameter as a function of time are plotted in Fig. 4. It can be pointed out that the slopes of these curves indicate the exponential growth rate of swell and neck diameter and their differences. However, Fig. 4 shows that the exponential growth rates of swell and neck are not constant; rather the exponential growth rate of their differences is constant. The same trend of the curves was found by the experiment of Goedde and Yuen ${ }^{8}$. At early time the deformation of swell is slower and gradually the slope of the curve increases though the maximum part of the curve is straight. The straight part of the curve for swell has maximum slope among the curves. The minimum slope of the curve for swell is $30 / \mathrm{sec}$ and the maximum of that is $91 / \mathrm{sec}$. The slow deformation of swell in early stage is due to the requirement of time for the propagation of surface tension force to the fluid motion. The opposite trend can be observed for the curve of neck, i.e. at early stage the deformation of neck is faster and later it becomes slower. The faster growth of neck is the consequence of the conservation of mass for the development of capillary wave. After some development of the neck, surface tension force becomes dominant due to small radius of curvature and consequently the growth of neck becomes slower. Up to the range of data plotted in Fig. 4 the maximum slope for the neck is $77 / \mathrm{sec}$ at the straight portion of the curve and the minimum is $34 / \mathrm{sec}$. In experiment, Goedde and Yuen ${ }^{8}$ found that the maximum slope of the curve for swell is $91 / \mathrm{sec}$ and that for neck is $78 / \mathrm{sec}$. Also the Fig. 4 shows that though the part of the curves for both swell and neck are straight, their slopes are higher than the slope of the curve plotted for the difference between swell and neck which can be found as $72 / \mathrm{sec}$. In experiment, it has been indicated as $74 / \mathrm{sec}$.

From the above comparison excellent agreement of the present numerics with the experimental data can be found. With the level of accuracy indicated by this comparison and discussion it can be concluded that the present numerical method is capable of capturing the capillary wave and can be a tool to investigate the phenomena of capillary instabilities. Therefore, the numerical method is then used to examine the detailed dynamics of a square cylindrical liquid column.

\section{Problem statement}

To understand the phenomena of capillary wave radiated from the tips of the liquid sheet during contraction, a numerical algorithm has been developed to solve time dependent two-dimensional Navier-Stokes equations with surface tension force. The algorithm can capture the capillary waves radiated on the surface of liquid sheet. The code is used to study the contraction of liquid sheet in an otherwise quiescent gas as well as the capillary waves formed on the liquid interfaces. Sulfur hexafluoride $\left(\mathrm{SF}_{6}\right)$ of critical temperature, $318.7 \mathrm{~K}$ and critical pressure, $3.76 \mathrm{MPa}$ is used as liquid. The thickness of the liquid sheet is $3.0 \times 10^{-4}$ and denoted as ' $b$ ' shown in Fig.5. The half length (L) and thickness ratio of the liquid sheet, $\mathrm{L} / \mathrm{b}$ is 40 . High length to thickness ratio is considered to keep the capillary waves away from the effects of boundary conditions imposed at the end of symmetry. The width of the sheet is long enough to solve the problem as two-dimensional. Gaseous nitrogen is used 


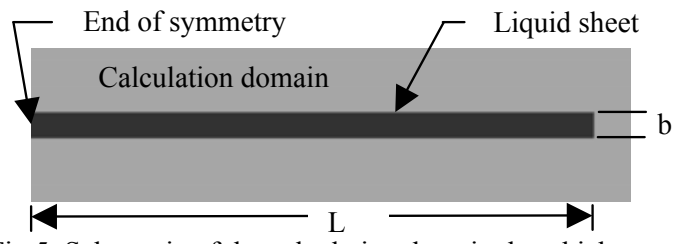

Fig.5: Schematic of the calculation domain; $b=$ thickness

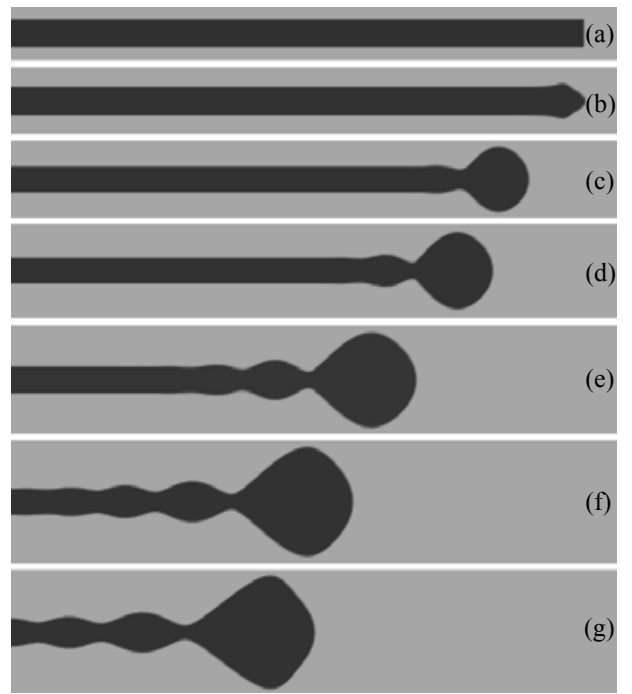

Fig.6: Evolution of capillary waves during contraction of liquid sheet; Dimensionless time, (a) $\mathrm{t}=0.0$, (b) $\mathrm{t}=0.32$, (c) $\mathrm{t}=2.54$, (d) $\mathrm{t}=4.0$, (e) $\mathrm{t}=7.0$, (f) $\mathrm{t}=10.0$, (g) $\mathrm{t}=13.0$.

as immiscible, viscous fluid with pressure of 7.0 MPa. The above numerical parameters are used for this calculation as some experimental results on liquid atomization problem are available in the work of Umemura ${ }^{17}$, co-author of this paper, using the same parameters.

\section{Capillary waves}

Figure 6 shows the time evolution history of capillary waves during the contraction of liquid sheet. The times reported have been made dimensionless with respect to the timescale, $t_{c}=\sqrt{\frac{\rho_{l} b^{3}}{\sigma}}$, where $\rho_{l}$ is the density of liquid, $\mathrm{b}$ the initial thickness of the liquid sheet chosen as the characteristic length scale and $\sigma$, the surface tension. Initially the tip of the liquid sheet is rectangular in shape. Due to surface tension force, the deformation of the shape is initiated at the rectangular corner of the tip. Initial deformation of the tip can be observed from Figs. 6(a and b). Three corners are generated from two corners of the rectangular shape (shown in Fig. 6(b)) and the deformation continues. Gradually, the tip of the liquid sheet becomes round with a neck to connect the head with the body of sheet. Due to surface tension, this neck initiates to form capillary waves on the rest of the liquid surface. After the formation of round end, the first wave can be observed at dimensionless time, 4.0 with amplitude of $0.14 \mathrm{~b}$ and the wavelength of $2.05 \mathrm{~b}$. At the same time the round shape of the liquid end becomes a wavy shape with amplitude of $0.61 \mathrm{~b}$ and wavelength of $3.02 \mathrm{~b}$. The wave propagates along the liquid surface to the midsection of the liquid creating waves of shorter wavelength with the distance away from the tip. The waves are stocking in nature and for time being they increase their wavelengths occupying more liquid.
Figures $6(\mathrm{e} \sim \mathrm{g})$ are of same interval of time showing the number of waves radiated on the liquid interfaces. By investigation it can be understood that the frequency of wave generation is not constant. The propagation time of the following waves is shorter than the predecessor which is the characteristics of capillary wave. It can be pointed out that Fig. 6(g) occupies four waves besides large tip wave. Due to space constraint Fig. 6(g) covers only two and a half waves showing the distance of liquid tip position from its original position at time, $t=0$. All the waves radiated in time, $t=13.0$ can be found in Fig. 7. Due to the end effect on surface tension, the shape of tip wave is different from the others. For easy understanding about the waves, the narrower and wider passages of the waves are denoted as "trough" and "peak", respectively as shown in Fig. 7. The position of different troughs from left boundary of calculation domain is mentioned in this figure. It is noted that the length of liquid sheet at time, $t=0$ is $12.0 \mathrm{~mm}$. At $\mathrm{t}=13.0$, after contraction, the length of the sheet becomes $8.38 \mathrm{~mm}$ and within this time 5 complete waves are formed with different wave amplitudes. The peaks of the generating waves are asymptotic approach, i.e. the distance from trough to peak of the following wave decreases asymptotically. The half of this distance is considered as the amplitude of the wave. Accordingly the amplitude of the tip wave is $0.33 \mathrm{~mm}$ and that of the following four waves are 0.072, 0.038, 0.02 and 0.012 , respectively.

Figure 8 shows the variation of pressure along the centerline of liquid sheet. The pressure at different position of capillary waves can be understood from this figure. From Fig. 8 it can be understood that the pressure is high at the peaks and low at the troughs. The wave at the tip shows the highest pressure and gradually the peak values of both high and low pressures decrease in the waves away from the tip wave. Two effects of surface tension on pressure can be observed, which can be explained by considering the Fig. 8. At the troughs, the direction of surface tension force is toward the outside of the liquid, which causes low pressure. At the peaks, the surface tension force is activated toward inside of the liquid which causes high

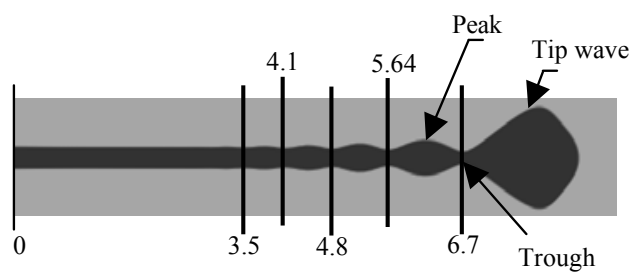

Fig.7: Position of capillary waves at time, $\mathrm{t}=13.0$. Numerical values indicate distance in $\mathrm{mm}$.

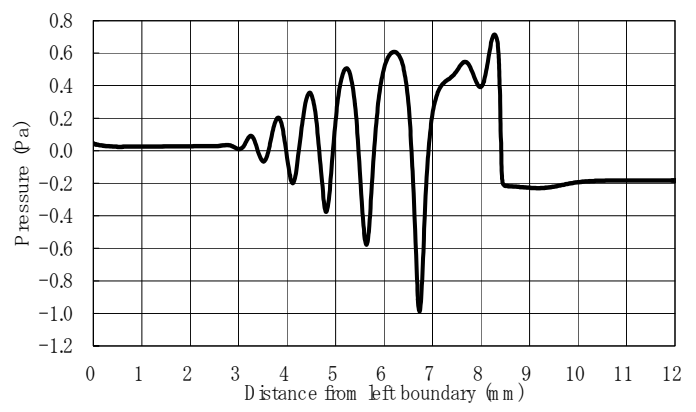

Fig. 8: Pressure distribution along the centerline of the liquid sheet at time, $\mathrm{t}=13.0$ 
pressure. Figure 9 shows that the distribution of $U$ velocity, the velocity along the direction of longitudinal axis of liquid sheet. The U-velocity towards right is considered as positive and that towards left is considered as negative. From Fig. 9 it can be found that the U-velocity increases at both of peaks and troughs, but in opposite direction, which means that the peak value of positive direction occurs at the troughs and that of negative direction occurs at the peaks of the waves. The highest peak velocity towards negative direction occurs in tip wave, which is caused by the surface tension force and the effect of liquid sheet contraction. It can be pointed out that the velocity is initiated by pressure and the flow occurs from high pressure region to low pressure region. Also in the developed flow field, this trend can be found in tip wave. In other region, the fluid velocity is motivated by recirculation as shown in Fig. 10. This figure shows a part of velocity vector field in magnified scale including only one wave at time, $t=13.0$. The length of vector shows the magnitude of the velocity. A pair of recirculations in trough and peak of the wave can be observed. The recirculation in the trough is anticlockwise and small in size, whereas, that in the peak is clockwise and large in size. The effect of large recirculation on centerline velocity can be found in Fig. 9, which shows that the span of negative velocity is larger than that of positive velocity. The peak values of both low and high pressure gradually decrease with the increase of curvature at both of the troughs and peaks of the wave resulting in the effect of surface tension force. On the other hand, the peak values of positive and negative velocity decrease gradually with the increase of narrowness at the troughs due to the conservation of mass. The high negative velocity of gas in front of the liquid tip as shown in Fig. 9 is caused by the effect of liquid velocity in the tip wave. This high velocity of gas does not have considerable effect on pressure field.

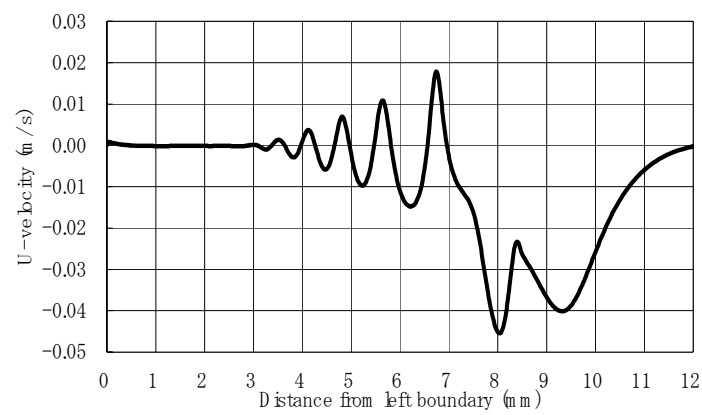

Fig. 9: U-velocity (velocity along the length of liquid sheet) distribution along the centerline of the liquid sheet at time, $\mathrm{t}=13.0$.

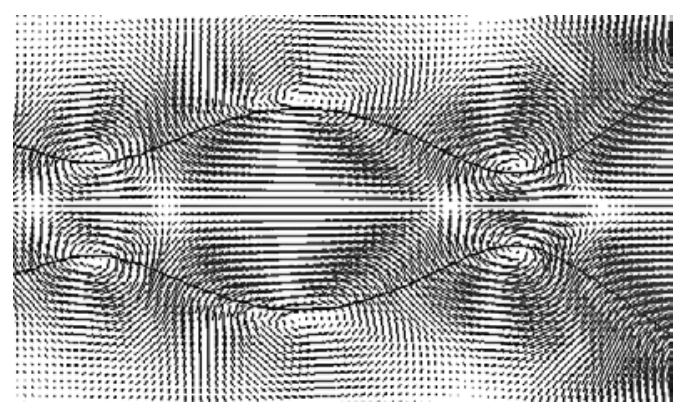

Fig. 10 A part of velocity vector field (magnified) including one wave at time, $\mathrm{t}=13.0$

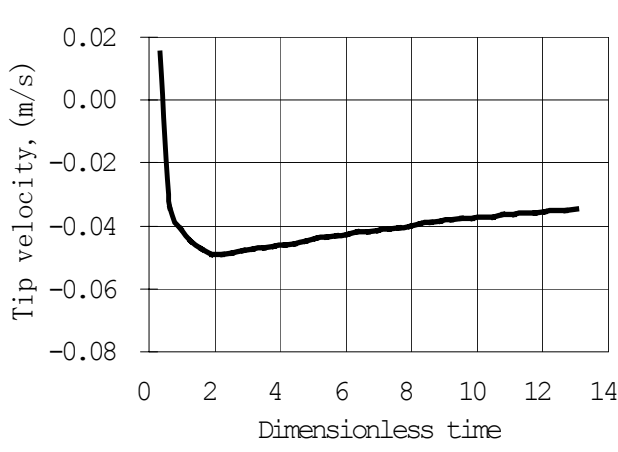

Fig. 11: Velocity of liquid sheet tip with time.

\section{Contraction of liquid cylinder}

Contraction of liquid sheet with time can be observed in Fig.6. The initialization of contraction depends on the tip shape of liquid cylinder. Due to high surface tension force at the rectangular corner of liquid tip, positive velocity of tip along lengthwise direction occurs resulting in a triangular shape of liquid tip as shown in Fig. 6(b) and consequently the length of cylinder increases slightly. However, the deformation of the tip continues and a bulbous end forms with the contraction of liquid. Due to the trend of attaining an equilibrium spherical shape of liquid, at first a spherical shape of the end is formed with the contraction of liquid (shown in Fig. 6(c)). The tip velocity calculated by $\mathrm{dL} / \mathrm{dt}$ is reported in Fig. 11 which shows that the tip velocity is not uniform. Initially the velocity is positive which increases the sheet length and soon the contraction of sheet length starts and the magnitude of negative velocity (velocity towards left) increases. The highest velocity attains at about dimensionless time, $\mathrm{t}=2.0$ during the process of contraction. This is expected because at that initial stage, the tip attains spherical shape as shown in Fig. 6(c) containing much liquid within small interval of time. Later on, the tip velocity decreases slowly due to occupying large space under tip wave.

\section{CONCLUSIONS}

A numerical investigation is performed to study the characteristic phenomena of capillary wave on the surface of liquid sheet. A computational algorithm is developed which is suitable to capture the capillary wave on the liquid surface. The computational code is validated with the published experimental data. The agreement of numerical results with the experiment is good enough to use the code to study the capillary phenomena. Using this code a twodimensional calculation is performed to study the capillary phenomena on the surface of liquid sheet during its contraction. It is found that the capillary wave is formed by surface tension force initiated at the tip of the sheet. The rectangular shape of tip is transformed into a triangular shape and the capillary waves are formed with a bulbous end. The capillary wave propagates with an asymptotic approach along the height of wave peaks from the longitudinal axis of the sheet. The formation of following waves takes shorter interval of time than that of its former wave and also the wavelength of the propagating waves becomes shorter than that of the previous one. Pressure along the longitudinal axis depends on the surface tension and radius of curvature at the peak and trough of the waves. The peak of both low and high pressures occurs at the position of troughs and peaks, respectively. Reverse 
trend on the distribution of horizontal velocity along the longitudinal direction can be found, which shows that the peak value of horizontal velocity in positive direction occurs at the troughs and that in negative direction occurs at the peaks of the wave. Both the peak values of pressure and velocity decrease with the decrease of wave amplitude by increasing the radius of curvature and passage of liquid flow to satisfy the conservation law of mass. During contraction, the tip velocity of the liquid sheet is not uniform. Initially the velocity increases sharply, after sometime it remains constant for a while and then it decreases slowly which continues up to the end of calculation.

\section{ACKNOWLEDGEMENT}

The authors gratefully acknowledge the financial support of Japan Space Forum to conduct this research.

\section{REFERENCES}

1. Gueyffier, D., Li, J., Nadim, A., Scardovelli, R. and Zaleski, S., "Volume-of-Fluid Interface Tracking with Smoothed Surface Stress Methods for ThreeDimensional Flows", J. Computational Physics, 152, 1999, pp. 423-456.

2. Brackbill, J. U. Kothe, D. B. and Zemach, C., "A Continuum Method for Modeling Surface Tension”, J. Computational Physics, 100, 1991, pp. 335-354.

3. Hirt, C. W. and Nichols, B. D., "Volume of Fluid (VOF) Method for the Dynamics of Free Boundaries", J. Computational Physics, 39, 1981, pp. 201-225.

4. Welch, S. W. J. and Wilson, J., "A Volume of Fluid Based Method for Fluid Flows with Phase Change", J. Computational Physics, 160, 2000, pp. 662-682.

5. Rider, J. W. and Kothe, "Reconstructing Volume Tracking", J. Computational Physics, 141, 1998, pp. 112-152.

6. Ashgriz, N. and Poo, J. Y., "FLAIR: Flux LineSegment Model for Advection and Interface Reconstruction”, J. Computational Physics, 93, 1991, pp. 449-468.
7. Rayleigh, L., "On the Capillary Phenomena of Jets", Proceedings of the Royal Society of London, 29, 1879, pp. 71-97.

8. Goedde, E. F. and Yuen, M. C., "Experiments on Liquid Jet Instability", J. Fluid Mech., 40, part 3, 1970, pp. 495-511.

9. Donnelly, R. J. and Glaberson, W., "Experiments on the Capillary instability of a liquid Jet", Proceedings of the Royal Society of London, Series A, 290, 1966, pp. 547-556.

10. Stone, H. A., Bently, B. J. and Leal, L. G., "An Experimental Study of Transient Effects in the Breakup of Viscous Drop", J. Fluid Mech, 173, 1986, pp. 131-158.

11. Stone, H. A. and Leal, L. G., "Relaxation and Breakup of an Initially Extended Drop in an Otherwise Quiescent Fluid”, J. Fluid Mech, 198, 1989, pp. 399-427.

12. Eggers, J., "Universal Pinching of 3D Axisymmetric Free-Surface Flow", Physical Review Letters, 71 (21), 1993, pp. 3458-3460.

13. Eggers, J., "Theory of Drop Formation", Phys. Fluids, 7 (5), 1995, pp. 941-953.

14. Eggers, J. and Dupont, T. F., "Drop Formation in a One-Dimensional Approximation of the NavierStokes Equation", J. Fluid Mech., 262, 1994, pp. 205221.

15. Kowalewski, T. A., "On the Separation of Droplets from a Liquid Jet", Fluid Dynamics Research, 17, 1996, pp. 121-145.

16. Eggers, J., "Nonlinear Dynamics and Breakup of Free-Surface Flows", Review of Modern Physics, 69 (3), 1997, pp. 865-929.

17. Umemura, A. and Wakashima, Y., "Atomization Regimes of a Round Liquid Jet with Near-Critical Mixing Surface at High Pressure", Proceedings of the Combustion Institute, 29, 2002, pp. 633-640. 